\title{
Mental Health Concerns of Undergraduate and Graduate Students: Depression, Anxiety, Eating Concerns, and Substance Misuse
}

\author{
Joshua J. Matacotta ${ }^{1 *}$ \\ College of Health Sciences, Western University of Health Sciences, USA \\ *Correspondence: Joshua J. Matacotta, jmatacotta@westernu.edu
}

\begin{abstract}
This study investigated mental health concerns reported by undergraduate and graduate college students attending a public university. The sample consisted of 1,451 students (Mage=23.6 years) seeking campus-based counseling services. The results show that depression, anxiety, eating concerns, and substance misuse are the most cited mental health concerns impacting academics and overall wellbeing. Data reveal other factors contributing to mental health concerns in the college population. These include a history of self-harm behaviors or suicide, past experiences of trauma, and uncertainty about or delay in accessing supportive services.
\end{abstract}

Keywords: mental health, students, college counseling

\section{INTRODUCTION}

College counseling center clinicians continue to encounter anxiety and depression as the most common general or top concerns experienced by undergraduate and graduate students. College students are at increased risk for mental health concerns or health risk behaviors due to significant stressors during a developmental phase with multiple transitions and changes (Huang et al., 2018). Additionally, the lifetime prevalence rates of "threat to self" characteristics (e.g., non-suicidal self-injury, serious suicidal ideation, and suicide attempts) among students receiving clinical services have increased over the last decade. Data from the Centers for Disease Control and Prevention (CDC) indicate an upward trend in suicide rates among young adults over the past few decades (Miron et al., 2019). Undergraduate and graduate students are under considerable stress due to academics, personal obligations, and future-oriented concerns about economic stability and employability. The consequences of perceived stress and mental health concerns such as depression contribute to elevated suicide risk, especially in the college population (Hirsch et al., 2019).

The increasing demand for mental health services on college campuses and psychoeducation about responding to students in distress require collaborative efforts beyond the college counseling center, given the relationship between student mental health 
and academic success (Kalkbrenner et al., 2019). In response to growing concerns about collegiate mental health, digital mental health interventions have been developed, although their benefits require further examination (Lattie et al., 2019). College counseling leadership struggles to balance clinical services' demands with staff burnout in a climate with dwindling resources and staffing shortages (Fu \& Cheng, 2017). University faculty, staff, administration, and the community where colleges operate play critical roles in college student retention and appropriate referral of students in distress. Learning is hindered by mental health concerns, and it is critical to understand how best to support students.

The types of health-promotion programs offered in the collegiate setting vary significantly, and resources are often insufficient to meet the student body's mental health needs. College counseling centers serve increasing numbers of students but are operating under resource strain. A typical student complaint is the wait time before seeing a mental health specialist, especially for urgent but non-emergency-related issues (Lipson et al., 2019). Additionally, students are often referred off-campus for mental health services but encounter various barriers to access. When students can access and use mental health services, studies indicate they can better manage academic demands and stressors (Eisenberg et al., 2009). The primary goal of this study was to identify the most common mental health concerns in undergraduate and graduate students using college counseling services.

\section{METHOD}

\section{Participants and Procedures}

Data was collected from 1,451 college students (69.4\% female-identified) seeking counseling services at a public university counseling and psychological services center in the academic year 2014-2015. Students completed clinical assessment measures using a center-provided iPad at intake and then met with a staff psychologist for further assessment. Students are scheduled to return for weekly appointments when counseling services are indicated. Repeated assessment of psychological symptoms is conducted at subsequent appointments and documented in the electronic medical record (EMR) for review by the clinician to monitor progress toward treatment goals.

\section{Measures}

Two measures were used as part of the standard intake procedure used by all counseling center member institutions of the Center for Collegiate Mental Health (CCMH). The Standardized Data Set (SDS) and the 62-item Counseling Center Assessment of Psychological Symptoms (CCAPS-62) are completed at intake. The SDS represents a standardized set of questions typically asked of college students seeking mental health services. It serves as the primary source of information on demographics, mental health 
history, and the use of alcohol and other drugs in the college counseling center population. Additionally, students are asked to rate the impact of the presenting problem on academics.

The CCAPS-62 includes items that assess eight distinct areas related to psychological symptoms and distress. It has strong psychometric properties and provides regularly updated peer-based norms drawn from extensive samples. Current norms for the CCAPS-62 were drawn from a CCMH dataset using information from all 59,606 participating college students seeking services at 97 institutions. The eight CCAPS-62 subscales are depression, generalized anxiety, social anxiety, academic distress, eating concerns, family distress, hostility, and substance use.

\section{Data analysis}

CCAPS data was collected from a sample of 1,451 students with a mean age of 23.6 $(S D=10.9)$ measured at baseline intake. All CCAPS-62 items are scored on a five-point scale $(0=$ Not at all like me, 1, 2, 3, $4=$ Extremely like $m e)$. As indicated in the CCMH CCAPS Technical Manual, raw scores were converted to CCAPS standardized scores to determine percentile ranking with the normative sample derived from a clinical setting. Percentiles are advantageous over raw or standardized scores for interpretation in clinical settings. For example, a percentile score of 80 on the depression subscale can be interpreted to mean that the student's self-reported scores are greater than $80 \%$ of students in the normative sample. The statistical package SPSS 25 was used to perform the statistical analysis. Frequencies were analyzed, and correlational analyses were performed using a two-tailed bivariate Pearson correlation coefficient. The top mental health concerns are evaluated and included in the analysis.

\section{Results}

Demographic data is outlined in Table 1. More than half (55\%) of students seeking counseling services reported experiencing at least one traumatic event in their life, of which $14 \%$ reported experiencing trauma within a year from their intake. Without assessing frequency or severity, $28 \%$ of the students indicated a history of abuse in their family. $41 \%$ of students reported prior utilization of mental health services. 231 students had first contact with the counseling center through triage (crisis) or urgent walk-in visit, of which 37 of these students were graduate students. 26 out of 258 students who answered the intake question about suicide attempt history reported a history of one or more suicide attempts. 
Table 1. Demographic Data

\begin{tabular}{|l|c|c|}
\hline Characteristic & N & $\mathbf{\%}$ \\
\hline Ethnicity & & \\
\hline American Indian/Alaskan Native & 10 & .7 \\
\hline Asian-Indian & 1 & .1 \\
\hline Asian-American & 279 & 19.2 \\
\hline Black/African American & 48 & 3.3 \\
\hline Filipino & 1 & .1 \\
\hline Hispanic-Mexican American & 5 & .3 \\
\hline Latinx/Spanish/Hispanic/Other & 520 & 35.8 \\
\hline Pacific Islander - Hawaiian & 16 & 1.1 \\
\hline White-Not of Hispanic Origin & 414 & 28.5 \\
\hline Decline/No Response & 51 & 3.5 \\
\hline & & \\
\hline Academic Level & & \\
\hline Freshman & 132 & 9.1 \\
\hline Sophomore & 165 & 11.4 \\
\hline Junior & 327 & 22.5 \\
\hline Senior & 626 & 43.1 \\
\hline Graduate & 180 & 12.4 \\
\hline Unknown & 21 & 1.4 \\
\hline Total & 1,451 & 100 \\
\hline
\end{tabular}

For each of the CCAPS-62 subscales, clinical cut-off points (low, mild, elevated) were established primarily to simplify interpretation and help distinguish nonclinical subscales scores from clinical scores (see Table 2). Although some scores are classified as "mild," clinicians view these as potentially problematic or mildly severe. Clinicians conducted further assessments to determine whether the score truly represents a clinical challenge. Scores above the higher cut-point do not indicate a diagnosable psychiatric condition but rather a need for further assessment and an increased likelihood of some clinical problem in that subscale area.

Table 2. CCAPS-62 Cut Points

\begin{tabular}{|l|c|c|}
\hline Subscale & $\begin{array}{c}\text { Low Cut Point } \\
\text { Percentile }\end{array}$ & $\begin{array}{c}\text { Elevated Cut Point } \\
\text { Percentile }\end{array}$ \\
\hline Depression & 34 & 56 \\
\hline Generalized Anxiety & 41 & 58 \\
\hline Social Anxiety & 56 & 76 \\
\hline Eating Concerns & 63 & 81 \\
\hline Academic Distress & 45 & $70^{*}$ \\
\hline Substance Use & 62 & 80 \\
\hline
\end{tabular}

*Note: Elevated Cut Points for the subscale is distributionally based by taking the value at the $70^{\text {th }}$ percentile. The other cut points are empirically based. 


\section{Depression and Anxiety Top Concerns}

Depression and anxiety were the most common mental health concerns. The depression subscale score was higher than 55\% of student scores in the national normative sample. The generalized anxiety subscale score and the social anxiety subscale score were higher than $50 \%$ and $59 \%$ of students in the national normative sample, respectively. Three items on the depression subscale are worth closer evaluation. $27.4 \%$ of students had mild or elevated scores on the suicidal ideation item ("I have thought of ending my life"). $33.7 \%$ reported feeling "sad all the time," and 27.1\% reported feelings of worthlessness. These three items were statistically significant and positively correlated ( 0.01 level) with the CCAPS-62 academic distress item, "It's hard to stay motivated for my classes."

Social isolation can serve to worsen depressive symptoms. $54 \%$ of students reported some level of feeling isolated and alone. $30 \%$ of the student sample had clinically elevated scores on the "alone and isolated" item. This was highly correlated with students' overall distress index scores.

\section{Eating Concerns and Substance Use}

In the study sample, the eating concerns and the substance use subscale scores were significantly elevated and higher than $64 \%$ of students in the national normative sample. Students with elevated clinical scores on the eating concerns subscale reported dissatisfaction with body shape, overeating or dieting frequently, and feeling better when eating less. Additionally, $47 \%$ of students reported alcohol use frequency that indicated a mild or elevated clinical concern. Despite this concern, only $8 \%$ felt the need to reduce the frequency of their alcohol use, and 6\% said others expressed concerns about their alcohol use. Over $10 \%$ of the sample indicated using marijuana three or more times within two weeks of their intake appointment. The most cited reasons for cannabis use included relaxation, sleep, socializing, and decreasing anxiety.

\section{Discussion}

This institutional study suggests that mental health concerns persist in the college population, and demand for counseling services remains high. Depression and anxiety exacerbate perceived stress related to academic success and future-oriented fear and uncertainty. The risk for self-harm behaviors increases when mental health concerns are not addressed, especially in students who isolate from family and friends. This study builds on prior studies investigating the ongoing demand for clinical services that address mental health issues in the young adult population (Duffy et al., 2019; Oswalt et al., 2020). Without adequate supportive services, students may turn to maladaptive coping behaviors with adverse health and academic outcomes. Students with unaddressed mental health concerns are more likely to terminate their academic studies prematurely and are at risk for future health-related problems. Further, it can be difficult for some students to resume their education later. Students who exit their educational institutions may lose access to support resources, supportive faculty and staff, and campus-based clinical services. 
With demand outpacing supply, an upward trend persists in mental health service utilization. This study confirms that depression and anxiety continue to be common mental health concerns for the college population. Symptoms of depression and anxiety are exceptionally high in incoming first-year students, outgoing seniors, and graduate students. Beyond the clinical symptoms, the data from this study reveal other factors contributing to mental health concerns in the college population. Among these are a history of self-harm behaviors or suicide, experiences of trauma, and uncertainty in decisions regarding the use of mental health services. Many students do not utilize supportive resources or counseling services until an unmanageable crisis has emerged. Psychoeducation, building coping strategies, and efforts to eliminate stigma about mental illness and accessing mental health services will serve students well when these prevention approaches are implemented on campus.

Substance misuse is an ongoing reported concern in the college student population. Substance misuse often results in negative academic consequences, a higher risk of unemployment after graduation, poor health outcomes, and potential injury or death (Bolin et al., 2017; Welsh et al., 2019). In fact, substance use has increased and is frequently cited as a maladaptive coping behavior utilized by students in response to stress or social anxiety (2017). While research on college substance and alcohol use is limited and inconsistent, this study confirms that students continue to use alcohol or other substances to manage anxiety and stress, despite years of efforts to identify education and prevention approaches to reduce these maladaptive coping behaviors.

Finally, body image and eating concerns were indicated as significant mental health concerns in those students citing eating concerns as a presenting problem. This is a growing concern in the population, and this study confirms that some students use food in response to distress or restrict food intake due to poor self-image. Obsessive food and dieting behaviors may not fully meet the diagnostic criteria for an eating disorder. However, these behaviors are often related to anxiety about meeting social standards of beauty and fitness. Issues related to body image and self-esteem often co-exist with other mental health concerns and substance misuse.

Larger scale studies are needed to further identify factors contributing to rising mental health concerns in the college population. A closer examination of student counseling services and specific interventions on emotional wellbeing and academic outcomes is needed. On-campus and community-based efforts to increase awareness about mental health concerns and university efforts to expand the capacity to provide mental health services will serve this population well. Prevention approaches and targeted interventions should address the top concerns of depression, anxiety, and substance misuse.

\section{Limitations}

One primary limitation of the study is its snapshot-in-time approach. This analysis did not include data from repeated assessment measures to examine psychological symptoms over time. There was no significance in interaction effects (gender, academic level). Although the sample size was sufficient for the study, findings are not entirely generalizable to the undergraduate and graduate student population. The reported mental health concerns are only those of students who voluntarily sought out support at their college counseling center. 


\section{References}

Bolin, R. M., Pate, M., \& McClintock, J. (2017). The impact of alcohol and marijuana use on academic achievement among college students. The Social Science Journal, 54(4), 430-437. https://doi.org/10.1016/j.soscij.2017.08.003

Duffy, M. E., Twenge, J. M., \& Joiner, T. E. (2019). Trends in Mood and Anxiety Symptoms and Suicide-Related Outcomes Among U.S. Undergraduates, 2007-2018: Evidence From Two National Surveys. Journal of Adolescent Health, 65(5), 590-598. https://doi.org/10.1016/j.jadohealth.2019.04.033

Eisenberg, D., Golberstein, E., \& Hunt, J. B. (2009). Mental Health and Academic Success in College. The B.E. Journal of Economic Analysis \& Policy, 9(1). https://doi.org/10.2202/1935-1682.2191

Fu, M., \& Cheng, A. W. (2017). College counseling services: Meeting today's demands. Psychological Services, 14(4), 403-406.

Hirsch, J., Rabon, J. K., Reynolds, E. E., Barton, A. L., \& Chang, E. C. (2019). Perceived stress and suicidal behaviors in college students: Conditional indirect effects of depressive symptoms and mental health stigma. Stigma and Health, 4(1), 98-106. https://doi.org/10.1037/sah0000125

Huang, J., Nigatu, Y. T., Smail-Crevier, R., Zhang, X., \& Wang, J. (2018). Interventions for common mental health problems among university and college students: A systematic review and meta-analysis of randomized controlled trials. Journal of Psychiatric Research, 107, 1-10. https://doi.org/10.1016/j.jpsychires.2018.09.018

Kalkbrenner, M. T., Jolley, A. L., \& Hays, D. G. (2019). Faculty Views on College Student Mental Health: Implications for Retention and Student Success. Journal of College 
Student Retention: Research, Theory \& Practice, 152102511986763. https://doi.org/10.1177/1521025119867639

Lattie, E. G., Adkins, E. C., Winquist, N., Stiles-Shields, C., Wafford, Q. E., \& Graham, A. K. (2019). Digital Mental Health Interventions for Depression, Anxiety, and Enhancement of Psychological Well-Being Among College Students: Systematic Review. Journal of Medical Internet Research, 21(7), e12869. https://doi.org/10.2196/12869

Lipson, S. K., Lattie, E. G., \& Eisenberg, D. (2019). Increased Rates of Mental Health Service Utilization by U.S. College Students: 10-Year Population-Level Trends (2007-2017). Psychiatric Services, 70(1), 60-63. https://doi.org/10.1176/appi.ps.201800332

Miron, O., Yu, K.-H., Wilf-Miron, R., \& Kohane, I. S. (2019). Suicide Rates Among Adolescents and Young Adults in the United States, 2000-2017. JAMA, 321(23), 2362-2364. https://doi.org/10.1001/jama.2019.5054

Oswalt, S. B., Lederer, A. M., Chestnut-Steich, K., Day, C., Halbritter, A., \& Ortiz, D. (2020). Trends in college students' mental health diagnoses and utilization of services, 2009-2015. Journal of American College Health, 68(1), 41-51. https://doi.org/10.1080/07448481.2018.1515748

Welsh, J. W., Shentu, Y., \& Sarvey, D. B. (2019). Substance Use Among College Students. FOCUS, 17(2), 117-127. https://doi.org/10.1176/appi.focus.20180037 